\title{
THE INFLUENCE OF THE DISSIPATIVE PART OF A GENERAL MARKOV PROCESS
}

\author{
R. V. CHACON
}

0. Introduction. In this paper we give a complete solution of a problem posed by $\mathrm{E}$. Hopf concerning the influence of the dissipative part of a general Markov process. Let $X$ be a space with points $x, y, \cdots$, and subsets $A, B, \cdots$, which form a field $\mathcal{F}$ and $\left\{P^{n}(x, A), n \geqq 1, x \in X, A \in \mathcal{F}\right\}$ and transition probabilities, of a general Markov process. They will be supposed to be countably additive in $A, \mathcal{F}$-measurable in $x$ and to satisfy the Chapman-Kolmogorov equation

$$
P^{n+1}(x, A)=\int_{X} P^{1}(x, A) P^{n}(x, d y) .
$$

The transition probabilities define related operators in various Banach spaces. We define for the Banach space of finite measures $m$ the transformation $T$ by the equation

$$
\operatorname{Tm}(A)=\int_{\mathbf{X}} P^{1}(x, A) m(d x) .
$$

We suppose that there exists a positive measure $m^{\prime}$ on $\mathcal{F}$ such that the set $M_{m^{\prime}}$ of $m^{\prime}$-absolutely continuous measures is mapped into itself. It is known and trivial that this is essentially no restriction to impose on $T$. Let $L_{1}$ be the Banach space of all $m^{\prime}$ integrable functions, and define a mapping $L^{*}$ of $L_{1}$ into itself by means of the following equation

$$
T m^{\prime \prime}(A)=\int_{\Delta} L^{*} f m^{\prime}(d x)
$$

if

$$
m^{\prime \prime}(A)=\int_{A} f m^{\prime}(d x) .
$$

$L^{*}$ is clearly positive and has norm less than or equal to one.

It is known [2, Theorem 8.1] that $X$ splits into two disjoint sets $C$ and $D, X=C+D$, the conservative and the dissipative part, respectively, of $X$, such that if $p_{1}$ is in $L_{1}$, is non-negative, then

Received by the editors November 2, 1959 and, in revised form, February 8, 1960. 


$$
\sum_{0}^{\infty} L^{* k} p_{1}(x)<\infty,
$$

for almost all $x$ in $D$,

and such that if $p_{2}$ is in $L_{1}$, is positive, then

$$
\sum_{0}^{\infty} L^{* k} p_{2}(x)=\infty, \quad \text { for almost all } x \text { in } C .
$$

We next consider the operations defined by

$$
\begin{aligned}
& L_{C f}^{*}=e_{C} L^{*} f, \\
& L_{D}^{*} f=e_{D} L^{*} f,
\end{aligned}
$$

where $e_{C}$ and $e_{D}$ are the characteristic functions of $C$ and of $D$, respectively. It can also be shown [2, Theorem 8.2] that $L_{D}^{*} L_{C}^{*}=0$, but that in general the product of the operators in reverse order does not vanish. We introduce further notation:

$$
\begin{aligned}
L_{D, n}^{*} & =\sum_{0}^{n-1} L_{D}^{* k}, \\
M_{n}^{*} & =L_{C}^{*} L_{D, n}^{*}, \\
L_{n}^{*}(f, p) & =\sum_{0}^{n-1} L^{* k} f / \sum_{0}^{n-1} L^{* k} p .
\end{aligned}
$$

$M_{n}^{*}$ measures the $f$ contribution to $C$ accumulated in $n$ successive trials. It may easily be shown that $M_{\infty}^{*} f=\lim _{n \rightarrow \infty} M_{n}^{*} f$ exists almost everywhere and is in $L_{1}$.

Hopf $\left[2\right.$, p. 44] asks the following question: is the limit of $L_{n}^{*}\left(f-f^{\prime}, p\right)$ (as $n$ tends to infinity) zero almost everywhere on $C$, where $f^{\prime}=M_{\infty}^{*} f$ ? We answer this question affirmatively. This means that in considering the limit of $L_{n}^{*}(f, p)$ we may replace $f$ by $f^{\prime}$ and $p$ by $p^{\prime}$ and thus obtain a separation of the conservative and dissipative parts of $X$.

1. Results and proofs. We state first the following lemmas:

LeMma 1. If $L^{*}$ is a positive linear operator of $L_{1}$ into $L_{1}$ such that its norm is less than or equal to one, and if we define $L^{*}(f, p)$ $=\lim _{n \rightarrow \infty} L_{n}^{*}(f, p)$ for $f$ and $p$ in $L_{1}$ and $p$ positive, then $L^{*}(f, p)$ is well defined, and if $\left\{f_{n}\right\}$ is a sequence of functions tending to zero in the $L_{1}$ norm, then the measure of the set where

$$
L^{*}\left(f_{n}, p\right) \geqq a
$$

tends to zero as $n$ tends to infinity, for each $a>0$. 
Proof. That $L^{*}(f, p)$ is well defined follows from [1, Theorem 1]. That the last property is satisfied follows from Hopf's maximal ergodic theorem, that

$$
\int_{A_{n}} f_{n} m^{\prime}(d x) \geqq a \int_{A_{n}} p m^{\prime}(d x)
$$

where $A_{n}=\left\{x: \sup _{k \geq 1} L_{\mathbf{k}}^{*}\left(f_{n}, p\right) \geqq a\right\}$.

LemMa 2. If $L^{*}$ is as in Lemma 1.1 and if $f$ and $p$ are in $L_{1}$ and $p$ is positive, and if we define $g=f-L^{* i} f$ for some fixed $i$, then

$$
\lim _{n \rightarrow \infty} L_{n}^{*}(g, p)=0
$$

almost everywhere on $C$.

Proof. We may suppose without loss of generality that $f$ is nonnegative. It follows from Lemma 1 of (1) that

$$
\lim _{n \rightarrow \infty} \frac{L^{*_{n+i f}}}{f+\cdots+L^{*_{n} f}}=0
$$

almost everywhere on the set $B=C \cap\left\{x: \sum_{0}^{\infty} L^{* k} f=\infty\right\}$. From this it follows that for each fixed $i$,

$$
\lim _{n \rightarrow \infty} L_{n}^{*}\left(L^{* i} f, f\right)=1
$$

almost everywhere on $B$. The proof of the lemma is complete on noting that on $C, p+\cdots+L^{*_{n}} p$ tends to infinity almost everywhere as $n$ tends to infinity, and thus that the lemma is trivial on that part of $C$ where $f+\cdots+L^{*_{n}} f$ does not tend to infinity.

THEOREM 1. If $L^{*}$ satisfies the conditions of Lemma 1, and if $f^{\prime}=M_{\infty}^{*} f$ and if $p$ is positive and if $f$ and $p$ are in $L_{1}$ then

$$
\lim _{n \rightarrow \infty} L_{n}^{*}\left(f-f^{\prime}, p\right)=0
$$

almost everywhere on $C$.

Proof. We note first that it follows directly from Lemma 2 that

$$
\lim _{n \rightarrow \infty} L_{n}^{*}\left(L^{* i} f, p\right)-L_{n}^{*}(\mathrm{f}, p)=0 \text { a.e. on } C \text {. }
$$

If we define $f_{i}=L_{C}^{*} \sum_{0}^{i-1} L_{D}^{* k} f+L_{D}^{* i} f$ we have, also from Lemma 2, that

$$
\lim _{n \rightarrow \infty} L_{n}^{*}\left(f_{i}, p\right)-L_{n}^{*}\left(L^{* i} f, p\right)=0 \text { a.e. on } C,
$$


because $f_{i}$ can be written as a sum of functions

$$
f_{i}=h_{0}+h_{1}+\cdots+h_{i}
$$

such that

$$
L^{*_{i} f}=L^{*_{i-1}} h_{0}+L^{*_{i-2}} h_{1}+\cdots+h_{i},
$$

and this means that the difference of (1.2) is equal to (in terms of the $\left.h_{j}\right)$

$$
\begin{aligned}
L_{n}^{*}\left(h_{0}, p\right)-L_{n}^{*}\left(L^{* i-1} h_{0}, p\right)+L_{n}^{*}\left(h_{1}, p\right)-L_{n}^{*}\left(L^{* i-2} h_{1}, p\right)+\cdots & \\
& +L_{n}^{*}\left(h_{i}, p\right)-L_{n}^{*}\left(h_{i}, p\right)
\end{aligned}
$$

and each difference tends to zero separately (the last, of course, equals zero) by Lemma 2 . To see that $f_{i}$ can be written as such a sum, note that since

$$
\begin{gathered}
f_{i}=L_{C}^{*} \sum_{0}^{i-1} L_{D}^{* k} f+L_{D}^{* i} f \\
L^{* i-(k+1)} L_{C}^{*} L_{D}^{* k} f=L_{C}^{* i-k} L_{D}^{* k} f,
\end{gathered}
$$

and $\left(L_{D}^{*} L_{C}^{*}=0\right)$

$$
L^{* i} f=\left(L_{C}^{*}+L_{D}^{*}\right)^{i} f=\sum_{k=0}^{i} L_{C}^{* i-k} L_{D}^{* k} f
$$

we may take $h_{j}=L_{C}^{*} L_{D}^{* j} f, j=0,1, \cdots, i-1$, and $h_{i}=L_{D}^{* i} f$. It follows easily from (1.1) and (1.2) that

$$
\lim _{n \rightarrow \infty} L_{n}^{*}\left(f_{i}, p\right)-L_{n}^{*}(f, p)=0
$$

almost everywhere on $C$ since the difference of (1.3) is equal to

$$
L_{n}^{*}\left(f_{i}, p\right)-L_{n}^{*}\left(L^{* i} f, p\right)+L_{n}^{*}\left(L^{* i} f, p\right)-L_{n}^{*}(f, p) .
$$

We may, and do, suppose that $f$ is non-negative, without loss of generality. We have $\left(L_{D}^{*} L_{C}^{*}=0\right)$ that

$$
L^{* k} L_{D}^{* i} f=\sum_{j=0}^{k} L_{C}^{* j} L_{D}^{* i+k-j} f
$$

and thus that

$$
\sum_{k=0}^{n-1} L^{* k} L_{D}^{* i} f=\sum_{k=0}^{n-1} \sum_{j=0}^{k} L_{C}^{* j} L_{D}^{* i+k-j} f
$$


Since

$$
\sum_{k=0}^{n-1} \sum_{j=0}^{k} L_{C}^{* j} L_{D}^{* i+k-j} f=\sum_{j=0}^{n-1} \sum_{k=j}^{n-1} L_{C}^{* j} L_{D}^{* i+k-j} f=\sum_{j=0}^{n-1} \sum_{k=0}^{n-(j+1)} L_{C}^{* j} L_{D}^{* i+k} f
$$

it follows that

$$
\sum_{k=0}^{n-1} L^{* k} L_{D}^{* i} f \leqq \sum_{j=0}^{n} \sum_{k=0}^{n-(j+1)} L_{C}^{* j} L_{D}^{* i+k} f
$$

and that on $C$,

$$
\sum_{k=0}^{n-1} L^{* k} L_{D}^{* i} f \leqq \sum_{j=0}^{n-1} L_{C}^{* j} \sum_{k=0}^{n-(j+1)} L_{C}^{*} L_{D}^{* i+k} f \leqq \sum_{j=0}^{n-1} L^{* j} \sum_{k=0}^{\infty} L_{C}^{*} L_{D}^{* i+k} f
$$

This implies that on $C$ we have

$$
L_{n}^{*}\left(L_{D}^{* i} f, p\right) \leqq L_{n}^{*}\left(\sum_{k=i}^{\infty} L_{C}^{*} L_{D}^{* k} f, p\right) .
$$

Now, it follows from (1.4), since $f^{\prime}=\sum_{k=0}^{\infty} L_{C}^{*} L_{D}^{* k} f$, that

$$
\begin{aligned}
\left|L_{n}^{*}\left(\jmath^{\prime}, p\right)-L_{n}^{*}\left(f_{i}, p\right)\right| & \leqq\left|L_{n}^{*}\left(\sum_{k=i}^{\infty} L_{C}^{*} L_{D}^{* k} f, p\right)-L_{n}^{*}\left(L_{D}^{* i} f, p\right)\right| \\
& \leqq 2 L_{n}^{*}\left(\sum_{k=i}^{\infty} L_{C}^{*} L_{D}^{* k} f, p\right)
\end{aligned}
$$

on $C$, and thus that

$$
\left|L^{*}\left(f^{\prime}, p\right)-L^{*}\left(f_{i}, p\right)\right|
$$

can be greater than a positive number $a$ on a subset of $C$ of measure which tends to zero as $i$ tends to infinity, by Lemma 1. We now note that

$$
L^{*}\left(f^{\prime}-f, p\right)=L^{*}\left(f^{\prime}, p\right)-L^{*}\left(f_{i}, p\right)+L^{*}\left(f_{i}, p\right)-L^{*}(f, p),
$$

and that the second difference is zero by (1.3) and that the first satisfies the condition listed above for (1.6). This clearly proves the theorem.

\section{BiblogRAPHy}

1. R. V. Chacon and D. S. Ornstein, $A$ general ergodic theorem, Illinois J. Math. vol. 4 (1960) pp. 153-160.

2. E. Hopf, The general temporally discrete Markoff process, J. Math. Mech. vol. 3 (1954) pp. 13-45.

UNIVERSITY OF WISCONSIN 